\title{
Earth rotation based on the celestial coordinates of the celestial intermediate pole
}

\section{The dynamical equations}

\author{
N. Capitaine ${ }^{1}$, M. Folgueira ${ }^{1,2}$, and J. Souchay ${ }^{1}$ \\ 1 Observatoire de Paris, SYRTE/UMR 8630-CNRS, 61 avenue de l'Observatoire, 75014 Paris, France \\ e-mail: capitain@danof.obspm.fr; Jean.Souchay@obspm.fr \\ 2 Instituto de Astronomía y Geodesia (UCM-CSIC). Facultad de Ciencias Matemáticas, Universidad Complutense de Madrid, \\ Ciudad Universitaria, 28040 Madrid, Spain \\ e-mail: martafl@mat.ucm.es
}

Received 6 July 2005 / Accepted 13 August 2005

\section{ABSTRACT}

\begin{abstract}
We present a study of the Earth's rotational motion in terms of the Earth Orientation Parameters (EOP) of the new paradigm that is recommended by the IAU 2000 resolutions to transform between the celestial and terrestrial reference systems. This paper presents the first part of the study whose purpose is to establish the dynamical equations of the rotation of a rigid Earth as a function of these new parameters. Starting from Euler dynamical equations for a rigid Earth, and using expressions for the components of the instantaneous rotation vector as functions of the celestial coordinates $X, Y$ of the Celestial intermediate pole (CIP) and of the Earth rotation angle (ERA), the equations of Earth rotation were obtained explicitly in terms of those parameters. Taking into account the order of magnitude of the terms of these equations, we obtain the most appropriate form of the equations for a practical integration. We then investigated the possible methods of integration for providing semi-analytical solutions for the $X$ and $Y$ variables in the axially symmetric case. We also perform a number of tests regarding the efficiency of these methods, based on the IAU 2000 precession-nutation. We extended this approach to a deformable Earth, based on integration constants compliant with the new $\mathrm{P} 03$ precession model.
\end{abstract}

Key words. astrometry - reference systems - ephemerides - times - celestial mechanics

\section{Introduction}

The nutation part of the IAU 2000A precession-nutation adopted by IAU 2000 Resolution B1.6 (IAU Transactions 2000) has been generated by Mathews et al. (2002) by the convolution of the MHB 2000 transfer function with the rigid Earth nutation REN-2000 of Souchay et al. (1999); it has been provided in terms of components $\Delta \psi$ and $\Delta \varepsilon$, respectively in longitude and obliquity.

The IAU 2000 resolutions have also recommended the use of the Celestial Intermediate Pole (CIP) and of the "new paradigm" to transform between the International Terrestrial System (ITRS) and the geocentric celestial reference system (GCRS).

The CIP has been defined by IAU 2000 Resolution B1.7, as the pole close to the instantaneous pole of rotation, the motion of which is specified in the GCRS by motion of the Tisserand mean axis of the Earth with periods greater than two days. Its motion in the GCRS therefore includes, by convention, all the nutations in space with periods greater than 2 days in the
GCRS, the nutations with periods less than 2 days being modeled by their equivalent polar motion with respect to the ITRS. Consequently, the GCRS motion of the CIP differs from that of the pole of the Earth's figure axis by those subdiurnal terms (see Capitaine 2000, 2002 for more details).

The new paradigm specified by IAU 2000 Resolution B1.8 to transform between the ITRS and GCRS is based on the Celestial and Terrestrial ephemeris origins, CEO and TEO, respectively", which are implementations of the "nonrotating origin" (Guinot 1979; Capitaine et al. 1986) in the GCRS and ITRS, respectively. It uses (i) the Earth rotation angle (ERA) and (ii) the position of the CIP both in the ITRS and GCRS. The ERA is the angle along the equator of the CIP between the CIO and the TIO. The position of the CIP in the ITRS is provided by the $x, y$-coordinates of the CIP unit vector in the ITRS, which are the usual "pole coordinates".

${ }^{1}$ Now called, according to the recommendations of the IAU Working Group on "Nomenclature for fundamental astronomy", the Celestial and Terrestrial "intermediate" origins, CIO and TIO, respectively; this will be used in this paper. 
The position of the CIP in the GCRS is provided by the $x, y$ coordinates of the CIP unit vector (i.e. direction cosines) in the GCRS, which are currently expressed in the form of $(X, Y)$ coordinates, in arcseconds, the values of which approximately represent the corresponding angles with respect to the polar axis of the GCRS. The CIP position $(X, Y)$ takes rigorous account of frame bias, precession and nutation (Capitaine 1990). These quantities represent the GCRS orientation of the celestial intermediate system in which astronomical Earth-based observations are naturally referred; they are, for example, the parameters to which VLBI is directly sensitive (Brzezinski \& Capitaine 1993; Capitaine et al. 2003b). Therefore theoretical expressions of these parameters allow one to compare theory and observation without any intermediary and thus to best estimate the basic physical parameters (e.g. the dynamical Earth's parameters).

The current IAU 2000 expressions for the $(X, Y)$ coordinates have been derived by Capitaine et al. (2003a) from the IAU 2000 expressions of $\Delta \psi$ and $\Delta \varepsilon$ variables. In this paper, we will use a different approach that will consist in developing the equations for Earth rotation using explicitly the $(X, Y)$ coordinates with the aim of providing the precession-nutation model directly in the form recommended by the IAU 2000 resolutions.

The equations of the rotational motion of the rigid Earth are based on the equations of angular momentum conservation that can be expressed in various forms. Existing analytical or semianalytical solutions for Earth rotation have been derived by solving either Euler equations (Woolard 1953; Bretagnon et al. 1997), or Hamiltonian equations (Kinoshita 1977; Souchay et al. 1999), or alternatively, the angular momentum equations themselves (Woolard 1953; Roosbeek \& Dehant 1998). Solutions are either for Euler angles of the terrestrial reference system with respect to the ecliptic system at a fixed epoch (Woolard 1953; Bretagnon et al. 1997), or for Andoyer variables and their canonically conjugate variables (Souchay et al. 1999). Different iterative approaches have been used to solve the equations. In some recent cases (Souchay et al. 1999; Roosbeek \& Dehant 1998) the solutions have first been obtained relative to the angular momentum axis and then transformed into solutions for the axis of figure by adding Oppolzer terms. In other cases, they have been directly referred to the axis of figure (Bretagnon et al. 1997) by solving second order differential equations by successive approximations.

Our purpose here is to establish variational equations for the rotational motion of the Earth in terms of the $(X, Y)$ coordinates of the CIP and ERA and to find a method to solve the system with a semi-analytical approach in the most accurate way. We therefore investigate the possible methods for providing semi-analytical solutions for the $X$ and $Y$ variables in the axially symmetric case, ensuring a microarcsecond accuracy over a time-span of a few hundred years. As the consideration of a rigid model is only the first step in solving the Earth's rotational equations, we also study how the equations in terms of the $X$ and $Y$ variables can be generalized to the case of a deformable body and how this will modify the solution.

However, the use of the $(X, Y)$ variables may not allow one to write the complete equations in a sufficiently compact form to provide rigorous analytical solutions in a simple and direct way. Therefore, in another paper (Folgueira et al. 2005) we will in parallel discuss the most suitable sets of variables (especially canonical variables) to be used for analytically integrating the equations of the Earth rotation for a rigid Earth, with the property of (i) being the closest as possible to the $(X, Y)$ coordinates and (ii) leading to equations that can be integrated in the simplest way to provide rigorous analytical solutions. This will allow us to evaluate the theoretical accuracy of the semianalytical solution that can be obtained in solving the equations for a rigid Earth established in this paper.

Moreover, in order to check the semi-analytical solution against a numerical solution based on the same models and constants, another paper (Souchay et al. 2005) will be devoted to solving the complete equations in terms of $(X, Y)$ and ERA, using numerical integration.

Once the comparison between these three independent approaches is completed, the final semi-analytical solution for a rigid Earth for the $X$ and $Y$ variables will be provided in a further paper (denoted Paper II in the following).

In Sect. 2, after having recalled the necessary definitions and background relative to the transformation between the celestial and terrestrial reference systems, we derive the expression of the components of the instantaneous rotation vector as functions of the celestial coordinates $X, Y$ of the CIP and the ERA. In Sect. 3, starting from Euler dynamical equations for a triaxial rigid Earth and using the expressions obtained in Sect. 2, we develop the Earth rotation equations as function of intermediary variables and, then in the axially symmetric case, in terms of the $X, Y$ parameters. Then, based on a numerical evaluation of the order of magnitude of the terms of these latter equations, we get their most appropriate form for a practical integration. In Sect. 4, we investigate the methods for solving the equations based on the method of variation of parameters. In Sect. 5, we describe the semi-analytical approach to be followed for providing semi-analytical solutions for $X$ and $Y$; we report on the tests that have been performed regarding the efficiency of our approach, based on the IAU 2000 precession-nutation. In Sect. 6, we investigate an extension of this approach to the more general model of a deformable Earth, compliant with the P03 precession of Capitaine et al. (2003b) that includes the contribution of the secular variation of the dynamical ellipticity of the Earth.

\section{Relations between the celestial and terrestrial reference systems and the Earth's instantaneous rotation vector}

The new paradigm to transform between the International Terrestrial System (ITRS) and the geocentric celestial reference system (GCRS) is based on the use of the GCRS $(X, Y)$ coordinates of the CIP, the ERA and the celestial intermediate reference system, denoted CIRS, defined by the CIP and the intermediate origin CIO. As the basic Euler equations of Earth rotation are expressed in terms of the ITRS components of the Earth's instantaneous rotation vector and as our purpose is to express these equations in terms of the GCRS $(X, Y)$ of the CIP, we should, in a first step, obtain the $(X, Y)$ expressions as functions of those components. We should also specify the 
relationships between the various reference systems that will be used in the different steps of this study.

\subsection{Transformation between the celestial and terrestrial reference systems}

The matrix that transforms from GCRS to ITRS can be written as (see IERS Conventions 2003) ${ }^{2}$ :

$$
[\mathrm{ITRS}]=W(t) R(t) C(t)[\mathrm{GCRS}]=M(t)[\mathrm{GCRS}],
$$

where $W(t), R(t), C(t)$ are the transformation matrices arising from polar motion, from the rotation of the Earth around the axis of the pole and from the motion of the celestial pole in the celestial system, respectively.

Two different representations can be used for the above transformation (for more details, see for example Capitaine 1990 or IERS Conventions 2003). In the classical paradigm, which is based on the use of the equinox as the origin along the equator of date, the GCRS to ITRS transformation, omitting polar motion, is written as the product of the individual rotation matrices $\mathbf{B}$ (bias) followed by $\mathbf{P}$ (precession) then $\mathbf{N}$ (nutation) and finally $\mathbf{T}$ (Earth rotation) expressed with Greenwich sidereal time. In the new paradigm, based on the use of the CIO as the origin along the equator of date, the same transformation is written as the product of a unique matrix $C(t)$ to deal with bias, precession and nutation, followed by a matrix $R(t)$ to deal with Earth rotation, expressed with ERA. The current explicit components of the matrix transformation $M(t)$ in (1), using the new representation, have the following form:

- $W(t)=R_{1}\left(-y_{\mathrm{p}}\right) R_{2}\left(-x_{\mathrm{p}}\right) R_{3}\left(s^{\prime}\right)$, $x_{\mathrm{p}}, y_{\mathrm{p}}$ being the ITRS coordinates of the CIP and $s^{\prime}$ the quantity which provides the ITRS position of the TIO;

- $R(t)=R_{3}(\theta)$,

$\theta$ being the Earth rotation angle (ERA) which is directly proportional to UT1. Note that the usual GST Greenwich sidereal time at date $t$ is related to ERA by subtracting the "equation of the origins", denoted $E O$ (i.e. GST $=\theta-E O$ ), that is the CIO right ascension of the equinox (see NFA IAU Working Group Recommendations 2005);

- $C(t)$ equals:

$$
C(t)=R_{3}(-s) \cdot\left(\begin{array}{ccc}
1-a X^{2} & -a X Y & -X \\
-a X Y & 1-a Y^{2} & -Y \\
X & Y & 1-a\left(X^{2}+Y^{2}\right)
\end{array}\right),
$$

$X, Y$ being the $x, y$ coordinates of the CIP unit vector in the GCRS, $s$ the quantity that provides the GCRS position of the CIO, $Z=\sqrt{1-\left(X^{2}+Y^{2}\right)}$ and $a=1 /(1+Z)$. Note that the celestial reference system derived from the GCRS by the matrix transformation $C(t)$ is the celestial intermediate system (CIRS) defined by the CIP equator and the CIO.

As we are interested here in developing the rotational equations of the Earth in terms of the $X, Y$ coordinates and the ERA, we will use the matrix transformation relative to the new paradigm.

\footnotetext{
${ }^{2}$ Note that the IERS Conventions is using the inverse ITRS-toGCRS transformation.
}

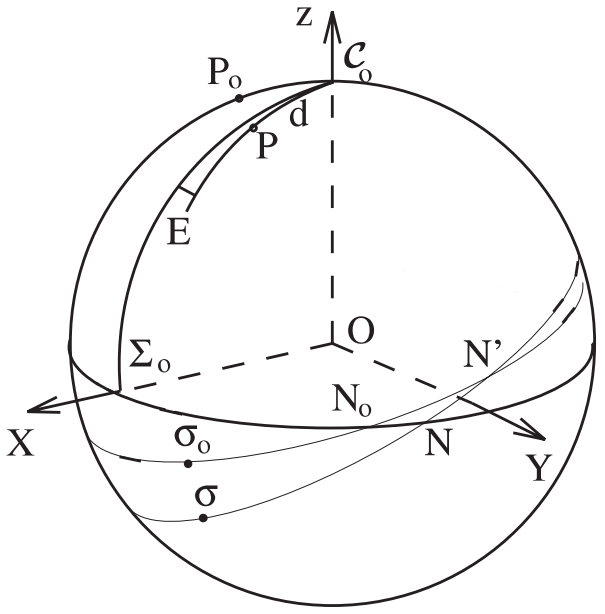

Fig. 1. The Geocentric celestial reference system, GCRS (Pole $C_{0}$, origin $\Sigma_{0}$ ) and the celestial intermediate system CIRS (CIP equator, origin $\sigma$ ). The CIP is represented on the figure by the point $\mathrm{P}$ whose GCRS coordinates are defined by: $X=\sin d \sin E, Y=\sin d \cos E$. The CIO is represented by the point $\sigma$; due to the kinematical definition of the NRO, $\sigma$ is such that at each instant its motion is perpendicular to the CIP equator. $P_{0}$ ans $\sigma_{0}$ are the CIP and CIO at J2000.

In order to simplify further developments for providing the expression of the instantaneous rotation vector, we will use an equivalent form of the above components of $M(t)$ by (i) replacing the matrix $R(t)$ by the rotation matrix $\mathbf{R}=R_{3}(\Theta)$ dealing with the total rotation about the $z$ axis and (ii) replacing the matrices $W(t)$ and $C(t)$ by $\mathbf{W}$ and $\mathbf{C}$, respectively deduced from $W(t)$ and $C(t)$ by omitting the $z$-rotation for the $s^{\prime}$ and $s$ quantities, respectively. This gives:

$\Theta=\theta-s+s^{\prime}$,

$\mathbf{M}=M(t)=\mathbf{W R C}$,

$\mathbf{W}=R_{1}\left(-y_{\mathrm{p}}\right) R_{2}\left(-x_{\mathrm{p}}\right)$,

$\mathbf{R}=R_{3}(\Theta)$,

$\mathbf{C}=\left(\begin{array}{ccc}1-a X^{2} & -a X Y & -X \\ -a X Y & 1-a Y^{2} & -Y \\ X & Y & 1-a\left(X^{2}+Y^{2}\right)\end{array}\right)$

The celestial reference system derived from the GCRS by the matrix transformation $\mathbf{C}$ is defined by the CIP equator and the origin $\Sigma$ on this equator. The CIP and the origins CIO and $\Sigma$ on the CIP equator are shown in Figs. 1 and 2, respectively.

\subsection{Expression of the instantaneous rotation vector as functions of the intermediate parameters}

The components $\left(\omega_{1}, \omega_{2}, \omega_{3}\right)$ of the Earth's instantaneous rotation vector $\vec{\omega}$ with respect to the ITRS can be expressed as follows (Kinoshita et al. 1979):

$\dot{\mathbf{M}} \mathbf{M}^{-1}=[\vec{\omega}]=\left(\begin{array}{ccc}0 & \omega_{3} & -\omega_{2} \\ -\omega_{3} & 0 & \omega_{1} \\ \omega_{2} & -\omega_{1} & 0\end{array}\right)$ 


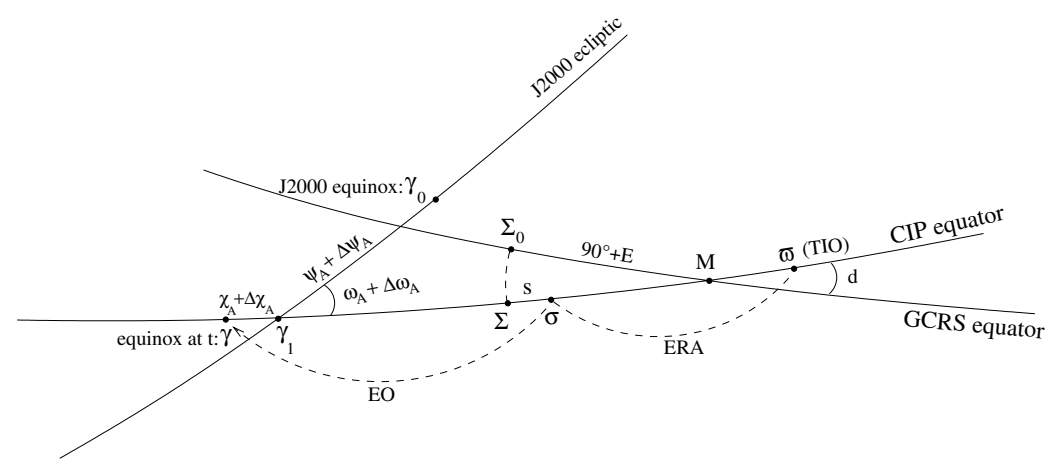

Fig. 2. The origins $\sigma$ (the CIO) and $\Sigma$ on the CIP equator and the Earth rotation angle (ERA) (i.e. $\theta$ ); $\gamma$ and $\gamma_{1}$ are the ascending nodes of the ecliptic of date and J2000 ecliptic, respectively on the CIP equator.

where $\dot{\mathbf{M}}$ and $\mathbf{M}^{-1}$ are respectively the derivative and the inverse of the matrix $\mathbf{M}$. The substitution of the relation (3) into (7) gives (Loyer 1997; Mathews 2003):

$$
\begin{aligned}
{[\vec{\omega}] } & =\dot{\mathbf{W}} \mathbf{W}^{-1}+\mathbf{W} \dot{\mathbf{R}} \mathbf{R}^{-1} \mathbf{W}^{-1}+\mathbf{W} \mathbf{R} \dot{\mathbf{C}} \mathbf{C}^{-1} \mathbf{R}^{-1} \mathbf{W}^{-1} \\
& =\left[\vec{\omega}_{W}\right]+\left[\vec{\omega}_{R}\right]+\left[\vec{\omega}_{C}\right]
\end{aligned}
$$

where:

$$
\begin{aligned}
& \vec{\omega}_{W}=\left(\begin{array}{c}
-\cos x_{\mathrm{p}} \dot{y}_{\mathrm{p}} \\
-\dot{x}_{\mathrm{p}} \\
\sin x_{\mathrm{p}} \dot{y}_{\mathrm{p}}
\end{array}\right), \quad \vec{\omega}_{R}=\left(\begin{array}{c}
\sin x_{\mathrm{p}} \cos y_{\mathrm{p}} \dot{\Theta} \\
-\sin y_{\mathrm{p}} \dot{\Theta} \\
\cos x_{\mathrm{p}} \cos y_{\mathrm{p}} \dot{\Theta}
\end{array}\right), \\
& \vec{\omega}_{C}=\frac{\mathbf{W R}}{Z}\left(\begin{array}{c}
-\dot{Y}-X \dot{s} \\
\dot{X}-Y \dot{s} \\
Z \dot{s}
\end{array}\right) \text {, }
\end{aligned}
$$

and $\dot{s}=a(Y \dot{X}-X \dot{Y})$.

For simplifying the equations, we can state, by convention, that the whole motion of the pole is modelled in the GCRS so that there is no motion of the pole in the ITRS (i.e. $x_{\mathrm{p}}=y_{\mathrm{p}}=s^{\prime}=0$; $\mathbf{W}=\mathbf{I})$. This in fact means that the $X, Y$ coordinates we are considering are not those of the CIP itself, but correspond to a pole $\mathrm{P}^{*}$, that is slightly different from the CIP in the sense that both (i) its free motion (i.e. Euler motion) and (ii) its subdiurnal nutations in space, are considered to be in the GCRS instead of the ITRS. $\mathrm{P}^{*}$ is actually the Earth's pole of figure. This will have to be taken into account when writing the final form of the equations in terms of $X$ and $Y$ of the CIP so that the above motions in the GCRS should be subtracted in order to be considered instead in the ITRS. Until we reach this final step, the quantities $X, Y, Z$ and the corresponding quantity $s$ will be denoted $X^{*}, Y^{*}, Z^{*}$ and $s^{*}$, respectively to make clear that they are referred to the pole of figure instead of the CIP.

With such a convention (that will be justified later), $\vec{\omega}$ reduces to the form:

$\vec{\omega}=\left(\begin{array}{c}0 \\ 0 \\ \dot{\Theta}\end{array}\right)+\frac{\mathbf{R}}{Z^{*}}\left(\begin{array}{c}-\dot{Y}^{*}-X^{*} \dot{s}^{*} \\ \dot{X}^{*}-Y^{*} \dot{s}^{*} \\ Z^{*} \dot{s}^{*}\end{array}\right)$

\subsection{Relationships between the various reference systems used in this paper}

In Sects. 2.1 and 2.2, we have considered the celestial and terrestrial reference systems in which the Earth rotation parameters are originally defined. Some properties of these reference systems and of other reference systems that will be used in this study are summarized in the following as well as the relationships between these systems:

- the ITRS in which the classical Euler equations are written; the origin on the ITRS equator is denoted $\varpi_{0}$ (i.e. $\varpi$ on the CIP equator if the polar motion is omitted; see Fig. 2);

- the GCRS, which is the reference system defined by IAU 2000 Resolution B1.3 for expressing motions with respect to the geocenter; the pole of the GCRS is denoted $C_{0}$, and the origin on the GCRS equator is denoted $\Sigma_{0}$ (see Figs. 1 and 2);

- the J2000 equatorial reference system, defined by the $\mathrm{J} 2000$ pole, $P_{0}$ and the inertial mean equinox of $\mathrm{J} 2000, \gamma_{0}$;

- the J2000 ecliptic reference system defined by the J2000 ecliptic pole, $Q_{0}$ and $\gamma_{0}$; the inclination of the $\mathrm{J} 2000$ equator with respect to the $\mathrm{J} 2000$ ecliptic is $\epsilon_{0}$;

- the equatorial reference system, defined by the CIP (denoted P) and the intersection, $\gamma_{1}$ of the J2000 ecliptic and the CIP equator; the inclination of the CIP equator on the $\mathrm{J} 2000$ ecliptic is $\omega_{A}+\Delta \omega_{A}$, which is the accumulated precession and nutation in obliquity from $\mathrm{J} 2000$; $\gamma_{1}$ is distant (1) from $\gamma_{0}$ along the $\mathbf{J} 2000$ ecliptic by the quantity $\psi_{A}+\Delta \psi_{A}$, which is the accumulated precession and nutation in longitude from $\mathbf{J} 2000$, and (2) from the equinox $\gamma$ along the CIP equator by the quantity $\chi_{A}+\Delta \chi_{A}$, which is the accumulated precession of the ecliptic along the CIP equator from J2000 (Lieske et al. 1977);

- the celestial intermediate reference system (CIRS), defined by the CIP and the CIO (denoted $\sigma$ ); $\gamma_{1}$ is distant from the $\mathrm{CIO}$ along the equator of the CIP by the quantity $-E O+$ $\left(\chi_{A}+\Delta \chi_{A}\right), E O$ being the "equation of the origins", $\sigma \gamma$;

- the equatorial reference system defined by the CIP and the point $\Sigma$ on the equator of the CIP; $\Sigma$ is such that $\Sigma \mathrm{M}=\Sigma_{0} \mathrm{M}, \mathrm{M}$ being the node of the equator of the CIP on the GCRS equator; $\Sigma$ is distant from the CIO by the quantity $-s$. This celestial reference system will be denoted CIRS' in the following. 
Table 1. Transformation between the various reference systems used in this study. $(\psi, \omega, \phi)$ are the Euler angles between the J2000 ecliptic and the CIP equator. $\left(\psi_{A}, \omega_{A}, \chi_{A}\right)$ are the classical precession quantities defined by Lieske et al. (1977) and $\Delta \psi_{A}$ and $\Delta \omega_{A}$ are the nutations in longitude and obliquity, respectively, referred to the J2000 ecliptic.

\begin{tabular}{ccc}
\hline \hline $\begin{array}{c}\text { TRANSFORMATION } \\
\text { (Reference plane, equinox / departure point) }\end{array}$ & MATRICES & $\begin{array}{c}\text { SECTION / FORMULA } \\
\text { in this paper }\end{array}$ \\
\hline CIRS': (CIP equator, $\Sigma$ ) $\longrightarrow$ CIRS: (CIP equator, $\sigma)$ & $R_{3}(s)$ & Sect. 2 \\
SIRS: (CIP equator, $\sigma) \longrightarrow$ GCRS: (GCRS equator, $\left.\Sigma_{0}\right)$ & C $/(6)$ \\
GCRS: (GCRS equator, $\left.\Sigma_{0}\right) \longrightarrow$ ITRS: (ITRS equator, $\left.\varpi_{0}\right)$ & M = WRC & Sect. 2 / (3), (4), (5), (6) \\
\hline (CIP equator, $\left.\gamma_{1}\right) \longrightarrow$ (CIP equator, $\left.\Sigma\right)$ & $R_{3}\left(-E O+s+\chi_{A}+\Delta \chi_{A}\right)$ & Sect. 5 \\
\hline (ecliptic J2000, $\left.\gamma_{0}\right) \longrightarrow$ (ecliptic J2000, $\left.\gamma_{1}\right)$ & $R_{3}\left(\psi_{A}+\Delta \psi_{A}\right)=R_{3}(-\psi)$ & Sect. 5 \\
(ecliptic J2000, $\left.\gamma_{1}\right) \longrightarrow$ (CIP equator, $\left.\gamma_{1}\right)$ & $R_{1}\left(\omega_{A}+\Delta \omega_{A}\right)=R_{1}(-\omega)$ & Sect. 5 \\
$\left(\right.$ CIP equator,,$\left.\gamma_{1}\right) \longrightarrow($ CIP equator, $\varpi)$ & $R_{3}\left(\mathrm{GST}+\chi_{A}+\Delta \chi_{A}\right)=R_{3}(\phi)$ & Sect. 5
\end{tabular}

The transformations between these various reference systems are specified in Table 1. The links between the various origins are shown in Fig. 2.

\section{Rotational equations in terms of the celestial coordinates of the CIP}

\subsection{Equations for a rigid Earth expressed in the celestial reference system}

\subsubsection{Triaxial case}

The equations for Earth rotation can be written in the classical form of Euler dynamical equations in the terrestrial system:

$\left\{\begin{array}{l}A \dot{\omega}_{1}+(C-B) \omega_{2} \omega_{3}=\lambda \\ B \dot{\omega}_{2}+(A-C) \omega_{1} \omega_{3}=\mu \\ C \dot{\omega}_{3}+(B-A) \omega_{1} \omega_{2}=v\end{array}\right.$

where $\left(\omega_{1}, \omega_{2}, \omega_{3}\right)$ and $(\lambda, \mu, v)$ are the terrestrial components of the instantaneous rotation vector and of the external torque, respectively, and $A, B, C$ are the Earth's principal moments of inertia.

We introduce the intermediary variables $\varpi_{1}, \varpi_{2}, \varpi_{3}$ defined by:

$\varpi_{1}=\frac{-\dot{Y}^{*}-X^{*} \dot{s}^{*}}{Z^{*}} ; \quad \varpi_{2}=\frac{\dot{X}^{*}-Y^{*} \dot{s}^{*}}{Z^{*}} ; \quad \varpi_{3}=\dot{s}^{*}$,

(i.e. in a first approximation, $\varpi_{1}$ and $\varpi_{2}$ are the time derivatives of $-Y^{*}$ and $X^{*}$ respectively). They are directly linked to the terrestrial components of the instantaneous rotation vector by:

$\vec{\omega}=\left(\begin{array}{c}0 \\ 0 \\ \dot{\Theta}\end{array}\right)+\mathbf{R}\left(\begin{array}{c}\varpi_{1} \\ \varpi_{2} \\ \varpi_{3}\end{array}\right)=\left(\begin{array}{c}\cos \Theta \varpi_{1}+\sin \Theta \varpi_{2} \\ -\sin \Theta \varpi_{1}+\cos \Theta \varpi_{2} \\ \dot{\Theta}+\varpi_{3}\end{array}\right)$

or, equivalently:

$\left(\begin{array}{c}\omega_{1} \\ \omega_{2} \\ \omega_{3}\end{array}\right)=\left(\begin{array}{c}\cos \Theta \varpi_{1}+\sin \Theta \varpi_{2} \\ -\sin \Theta \varpi_{1}+\cos \Theta \varpi_{2} \\ \dot{\theta}\end{array}\right)$

which shows that $\varpi_{1}$ and $\varpi_{2}$ are the two first components of the instantaneous rotation vector in the celestial reference system denoted CIRS' (cf. Table 1), linked to the axis of figure and the origin $\Sigma$ on the instantaneous equator.

Relation (14) allows us to transform (11) into the following equations, at the first order in the triaxial parameter, $\varepsilon$ :

$$
\begin{aligned}
\bar{A} \dot{\varpi}_{1}+ & \left\{\bar{A} \dot{\Theta}+(C-\bar{A})\left(\dot{\Theta}+\varpi_{3}\right)\right\} \varpi_{2} \\
+ & \varepsilon(C-2 \bar{A})\left\{-\sin 2 \Theta \varpi_{1}+\cos 2 \Theta \varpi_{2}\right\}\left(\dot{\Theta}+\varpi_{3}\right)= \\
& \lambda \cos \Theta-\mu \sin \Theta+\varepsilon\{\lambda \cos \Theta+\mu \sin \Theta\} \\
\bar{A} \dot{\varpi}_{2}- & \left\{\bar{A} \dot{\Theta}+(C-\bar{A})\left(\dot{\Theta}+\varpi_{3}\right)\right\} \varpi_{1} \\
+ & \varepsilon(C-2 \bar{A})\left\{\cos 2 \Theta \varpi_{1}+\sin 2 \Theta \varpi_{2}\right\}\left(\dot{\Theta}+\varpi_{3}\right)= \\
& \lambda \sin \Theta+\mu \cos \Theta+\varepsilon\{\lambda \sin \Theta-\mu \cos \Theta\} \\
C(\ddot{\Theta}+ & \left.\dot{\varpi}_{3}\right)+\bar{A} \varepsilon\left\{\sin 2 \Theta\left(\varpi_{2}^{2}-\varpi_{1}^{2}\right)+2 \cos 2 \Theta \varpi_{1} \varpi_{2}\right\}=v
\end{aligned}
$$

where $\bar{A}=\frac{A+B}{2}$ and $\varepsilon=\frac{B-A}{A+B}$ whose numerical values are $\bar{A}=8.0102 \times 10^{37} \mathrm{~kg}^{2}$ and $\varepsilon=1.24841 \times 10^{-5}$, respectively (Folgueira \& Souchay 2005).

The components $(L, M, N)$ of the external torque in the celestial reference system CIRS' are such that:

$$
\begin{aligned}
L & =\lambda \cos \Theta-\mu \sin \Theta \\
M & =\lambda \sin \Theta+\mu \cos \Theta \\
N & =v .
\end{aligned}
$$

Using the above expression for the torque components, along with the relation $\dot{\theta}=\dot{\Theta}+\dot{s}$, Eqs. (15) can be written in the form:

$$
\left\{\begin{aligned}
\bar{A} \dot{\varpi}_{1} & +\{C \dot{\theta}-\bar{A} \dot{s}\} \varpi_{2} \\
& +\varepsilon(C-2 \bar{A})\left\{-\sin 2 \Theta \varpi_{1}+\cos 2 \Theta \varpi_{2}\right\} \dot{\theta}= \\
& L+\varepsilon\{\lambda \cos \Theta+\mu \sin \Theta\} \\
\bar{A} \dot{\varpi}_{2} & -\{C \dot{\theta}-\bar{A} \dot{s}\} \varpi_{1} \\
& +\varepsilon(C-2 \bar{A})\left\{\cos 2 \Theta \varpi_{1}+\sin 2 \Theta \varpi_{2}\right\} \dot{\theta}= \\
& M+\varepsilon\{\lambda \sin \Theta-\mu \cos \Theta\} \\
C \ddot{\theta}+ & \bar{A} \varepsilon\left\{\sin 2 \Theta\left(\varpi_{2}^{2}-\varpi_{1}^{2}\right)+2 \cos 2 \Theta \varpi_{1} \varpi_{2}\right\}=N .
\end{aligned}\right.
$$


Equations (17) are the rigorous dynamical equations of Earth rotation for a rigid triaxial Earth, at the 1st order in the triaxial parameter, $\varepsilon$, expressed in terms of the ERA and the variables $\varpi_{1}, \varpi_{2}$ that are related to the GCRS coordinates $X^{*}$ and $Y^{*}$ of the unit vector directed to the pole of figure by relation (10).

\subsubsection{Axially symmetric case}

The terms in Eqs. (17), that are multiplied by the factor of triaxiality $\varepsilon$, give rise only to short periodic terms of semidiurnal period in the GCRS. These terms are in fact not relevant when considering only the motion in space with periods greater than 2 days, which actually correspond to the definition of the CIP; there is moreover no coupling effects between the diurnal or semi-diurnal nutations and terms appearing at periods greater than 2 days. The triaxiality effect can therefore be omitted without modifying the solutions for the celestial motion of the CIP. Note that $\bar{A}$ is, in this case, equal to $A$.

This results in a simplification of the equations, corresponding to the "axially symmetric case", as:

$$
\left\{\begin{array}{l}
\dot{\varpi}_{1}+\left\{\frac{C}{A} \dot{\theta}-\dot{s}\right\} \varpi_{2}=\frac{L}{A} \\
\dot{\varpi}_{2}-\left\{\frac{C}{A} \dot{\theta}-\dot{s}\right\} \varpi_{1}=\frac{M}{A} \\
C \ddot{\theta}=N .
\end{array}\right.
$$

These equations are the rigorous dynamical equations of Earth rotation for a rigid Earth model in the axially symmetric case, expressed in the celestial reference system in terms of the ERA and the variables $\varpi_{1}$ and $\varpi_{2}$.

For rotational symmetry, we know that: $v \equiv N=0$. Then, the third equation gives:

$\theta=\Omega t$,

$\Omega$ being the mean angular velocity of the Earth.

In the case of rotational symmetry where there is no subdiurnal nutations, the only difference of the pole $\mathrm{P}^{*}$ with respect to the CIP is in considering the free motion in the GCRS instead of the ITRS. We will state that the free motion corresponding to the following equations will be, at the final stage, taken into account as a motion in the ITRS so that the pole realized by the solutions will be the CIP. We will therefore omit the asterisk in denoting the quantities $X, Y, Z$ and $s$ in the following expressions and will use instead of relation (12), the following relation:

$\varpi_{1}=\frac{-\dot{Y}-X \dot{s}}{Z} ; \varpi_{2}=\frac{\dot{X}-Y \dot{s}}{Z} ; \varpi_{3}=\dot{s}$.

\subsection{The axially symmetric case: equations in terms of $X, Y$ and $E R A$}

\subsubsection{Equations in terms of $X, Y$ and $\dot{s}$}

The two first rotational equations for the rigid Earth in the axially symmetric case in terms of $X, Y, Z$ and $\dot{s}$ can be derived as follows from Eqs. (18), with $\theta=\Theta+s$, using expressions (20) for $\varpi_{1}$ and $\varpi_{2}$ :

$$
\begin{aligned}
& -\frac{1}{Z} \ddot{Y}-\frac{X}{Z} \ddot{s}-\frac{Y}{Z^{3}} \dot{Y}^{2}-\frac{C-A}{A} \frac{Y}{Z} \dot{s}^{2}-\frac{X}{Z^{3}} \dot{X} \dot{Y}-\frac{X Y}{Z^{3}} \dot{Y} \dot{s} \\
& -\frac{1}{Z}\left\{\frac{X^{2}}{Z^{2}}-\frac{C-2 A}{A}\right\} \dot{X} \dot{s}+\frac{C}{A} \frac{1}{Z} \dot{X} \dot{\Theta}-\frac{C}{A} \frac{Y}{Z} \dot{s} \dot{\Theta}=\frac{L}{A} \\
& \frac{1}{Z} \ddot{X}-\frac{Y}{Z} \ddot{s}+\frac{X}{Z^{3}} \dot{X}^{2}+\frac{C-A}{A} \frac{X}{Z} \dot{s}^{2}+\frac{Y}{Z^{3}} \dot{X} \dot{Y}-\frac{X Y}{Z^{3}} \dot{X} \dot{s} \\
& -\frac{1}{Z}\left\{\frac{Y^{2}}{Z^{2}}-\frac{C-2 A}{A}\right\} \dot{Y} \dot{s}+\frac{C}{A} \frac{1}{Z} \dot{Y} \dot{\Theta}+\frac{C}{A} \frac{X}{Z} \dot{s} \dot{\Theta}=\frac{M}{A} .
\end{aligned}
$$

\subsubsection{Equations in terms of $X, Y$ : rigorous form}

In the axially symmetric case, relations (2) and (19) give:

$\dot{\Theta}=\Omega-\dot{s}$.

We can introduce the following auxiliary quantities, related to the time derivatives of $Z, a$ and $\dot{s}$ :

$$
\begin{aligned}
\dot{Z}= & -\frac{1}{Z}\{X \dot{X}+Y \dot{Y}\} \\
\dot{a}= & \frac{a^{2}}{Z}\{X \dot{X}+Y \dot{Y}\} \\
\dot{s}^{2}= & a^{2} Y^{2} \dot{X}^{2}+a^{2} X^{2} \dot{Y}^{2}-2 a^{2} X Y \dot{X} \dot{Y} \\
\ddot{s}= & \frac{a^{2}}{Z} X Y \dot{X}^{2}+\frac{a^{2}}{Z}\left(Y^{2}-X^{2}\right) \dot{X} \dot{Y} \\
& -\frac{a^{2}}{Z} X Y \dot{Y}^{2}+a Y \ddot{X}-a X \ddot{Y} .
\end{aligned}
$$

The substitution of the above quantities and (22) for $\dot{\Theta}$ gives us the following equations in terms of $X$ and $Y$ :

$$
\left\{\begin{array}{l}
F_{1} \ddot{X}+F_{2} \ddot{Y}+F_{3} \dot{X}^{2}+F_{4} \dot{Y}^{2}+F_{5} \dot{X} \dot{Y}+F_{6} \dot{X}+F_{7} \dot{Y}=\frac{L}{A}, \\
G_{1} \ddot{X}+G_{2} \ddot{Y}+G_{3} \dot{X}^{2}+G_{4} \dot{Y}^{2}+G_{5} \dot{X} \dot{Y}+G_{6} \dot{X}+G_{7} \dot{Y}=\frac{M}{A},
\end{array}\right.
$$

where the expressions for the functions $F_{i}$ and $G_{i}$ (for $i=1$ to 7) are:

$$
\begin{aligned}
& F_{1}=-\frac{a}{Z} X Y \\
& F_{2}=-\frac{1}{Z}\left\{1-a X^{2}\right\} \\
& F_{3}=-\frac{a}{Z} Y\left\{\left(2-a Y^{2}\right)+\left(\frac{a}{Z}+\frac{1}{Z^{2}}\right) X^{2}\right\} \\
& F_{4}=\frac{Y}{Z}\left\{-\frac{1}{Z^{2}}+\left(\frac{a^{2}}{Z}+\frac{a}{Z^{2}}+a^{2}\right) X^{2}\right\} \\
& F_{5}=\frac{X}{Z}\left\{\left(2 a-\frac{1}{Z^{2}}\right)+\left(\frac{a^{2}}{Z}+\frac{a}{Z^{2}}\right) X^{2}-\left(\frac{a^{2}}{Z}+\frac{a}{Z^{2}}+2 a^{2}\right) Y^{2}\right\} \\
& F_{6}=\frac{C \Omega}{A} \frac{1}{Z}\left\{1-a Y^{2}\right\} \\
& F_{7}=\frac{C \Omega}{A} \frac{a}{Z} X Y
\end{aligned}
$$


and,

$$
\begin{aligned}
& G_{1}=\frac{1}{Z}\left\{1-a Y^{2}\right\} \\
& G_{2}=\frac{a}{Z} X Y=-F_{1} \\
& G_{3}=-\frac{X}{Z}\left\{-\frac{1}{Z^{2}}+\left(\frac{a^{2}}{Z}+\frac{a}{Z^{2}}+a^{2}\right) Y^{2}\right\} \\
& G_{4}=\frac{a}{Z} X\left\{\left(2-a X^{2}\right)+\left(\frac{a}{Z}+\frac{1}{Z^{2}}\right) Y^{2}\right\} \\
& G_{5}=\frac{Y}{Z}\left\{\left(\frac{1}{Z^{2}}-2 a\right)+\left(\frac{a^{2}}{Z}+\frac{a}{Z^{2}}+2 a^{2}\right) X^{2}-\left(\frac{a^{2}}{Z}+\frac{a}{Z^{2}}\right) Y^{2}\right\} \\
& G_{6}=\frac{C \Omega}{A} \frac{a}{Z} X Y=F_{7} \\
& G_{7}=\frac{C \Omega}{A} \frac{1}{Z}\left\{1-a X^{2}\right\} .
\end{aligned}
$$

We note that, except for the limitation to the axially symmetric case, no approximations have been made for deriving Eqs. (24) and expressions (25) and (26).

\subsubsection{Developed form of the coefficients $F_{i}$ and $G_{i}$}

In order to write Eqs. (24) in an appropriate form for being integrated, it is more convenient to have explicit formulas for $F_{i}$ and $G_{i}$ (for $i=1,7$ ) as functions of only the $X$ and $Y$ coordinates of the CIP in GCRS. As the aim is ensuring a microarcsecond accuracy over a time-span of a few hundred years, we have computed the Taylor series expansion of the quantities $a$ and $Z$ with respect to $X$ and $Y$, as in Capitaine (1990). After some developments, we obtain the following expressions for the $F_{i}$ and $G_{i}$ quantities, which have been verified to comply with the required accuracy:

$$
\begin{aligned}
& F_{1}=-\frac{1}{2} X Y-\frac{3}{8} X^{3} Y \\
& F_{2}=-1-\frac{1}{2} Y^{2}-\frac{3}{8} X^{2} Y^{2} \\
& F_{3}=-Y-\frac{3}{2} X^{2} Y-\frac{15}{8} X^{4} Y-\frac{1}{2} Y^{3} \\
& F_{4}=-Y-\frac{1}{2} X^{2} Y-\frac{3}{8} X^{4} Y-\frac{3}{2} Y^{3} \\
& F_{5}=-2 X Y^{2} \\
& F_{6}=\frac{C \Omega}{A}\left\{1+\frac{1}{2} X^{2}+\frac{3}{8} X^{4}+\frac{3}{8} X^{2} Y^{2}+\frac{5}{16} X^{6}\right\} \\
& F_{7}=\frac{C \Omega}{A}\left\{\frac{1}{2} X Y+\frac{3}{8} X^{3} Y+\frac{3}{8} X Y^{3}+\frac{5}{16} X^{5} Y\right\}
\end{aligned}
$$

and,

$$
\begin{aligned}
& G_{1}=1+\frac{1}{2} X^{2}+\frac{3}{8} X^{4}+\frac{3}{8} X^{2} Y^{2}+\frac{5}{16} X^{6} \\
& G_{2}=\frac{1}{2} X Y+\frac{3}{8} X^{3} Y \\
& G_{3}=X+\frac{3}{2} X^{3}+\frac{15}{8} X^{5}+\frac{1}{2} X Y^{2} \\
& G_{4}=X+\frac{1}{2} X^{3}+\frac{3}{8} X^{5}+\frac{3}{2} X Y^{2} \\
& G_{5}=2 X^{2} Y+3 X^{4} Y \\
& G_{6}=\frac{C \Omega}{A}\left\{\frac{1}{2} X Y+\frac{3}{8} X^{3} Y+\frac{3}{8} X Y^{3}+\frac{5}{16} X^{5} Y\right\} \\
& G_{7}=\frac{C \Omega}{A}\left\{1+\frac{1}{2} Y^{2}+\frac{3}{8} X^{2} Y^{2}\right\} .
\end{aligned}
$$

Equations (24), together with expressions (27) and (28), constitute the set of the differential equations for the Earth rotation parameters written in a compact and self-consistent form in terms of the $X, Y$ variables.

\subsubsection{Numerical estimation of the terms in the equations}

In order to investigate the possible methods for providing solutions of the above equations, we should know the order of magnitude of the various terms. Table 2 provides the estimated development of the terms of the differential equations as function of time based on the following approximate development for $X, Y$ and $s$ derived from Capitaine et al. (2003a):

$$
\begin{aligned}
X= & -0.02+2004.19 t-0.43 t^{2}-0.20 t^{3}-6.84 \sin \Omega \\
& +0.21 t \cos \Omega \\
Y= & +0.01-0.03 t-22.41 t^{2}+9.21 \cos \Omega+0.15 t \sin \Omega \\
s= & 0.004 t-0.04 t^{3}
\end{aligned}
$$

with the amplitudes in arcseconds rounded off to 10 mas and $t$ in century.

This table makes clear which terms are the prominent terms in the Earth's rotational equations expressed in terms of the $X, Y$ variables.

\subsubsection{Equations in terms of $X, Y$ : appropriate form for practical integration}

Table 2 shows that there are two prominent terms in each equation in (24), namely those multiplied by $F_{2}$ and $F_{6}$ for the first one, and those multiplied by $G_{1}$ and $G_{7}$ for the second one. Equations (24) can be transformed into a form where only these prominent terms are in the first member, the other terms, with 
Table 2. Numerical estimation of the terms of the differential equations (24) of Earth's rotation as function of time based on the IAU 2000 expressions of $X$ and $Y$ (Capitaine et al. 2003a).

\begin{tabular}{rrrrrr}
\hline \hline \multirow{2}{*}{ Term } & \multicolumn{5}{c}{ Coefficient (in arcseconds) of } \\
\cline { 2 - 6 } & $t^{0}$ & $t^{1}$ & $t^{2}$ & $t^{3}$ & $t^{4}$ \\
\hline$F_{1} \ddot{X}$ & - & $-0.9 \times 10^{-8}$ & $-0.1 \times 10^{-7}$ & $-0.2 \times 10^{-2}$ & $-0.2 \times 10^{-2}$ \\
$F_{2} \ddot{Y}$ & 44.8 & $-0.1 \times 10^{-1}$ & $0.2 \times 10^{-3}$ & $-0.1 \times 10^{-14}$ & $0.3 \times 10^{-6}$ \\
$F_{3} \dot{X}^{2}$ & $0.1 \times 10^{-7}$ & $-0.1 \times 10^{-10}$ & $0.2 \times 10^{-2}$ & $-0.2 \times 10^{-5}$ & $-1.0 \times 10^{-6}$ \\
$F_{4} \dot{Y}^{2}$ & - & - & $0.6 \times 10^{-11}$ & $-0.2 \times 10^{-14}$ & $0.1 \times 10^{-5}$ \\
$F_{5} \dot{X} \dot{Y}$ & - & - & $0.3 \times 10^{-20}$ & $-0.3 \times 10^{-23}$ & $0.1 \times 10^{-14}$ \\
$F_{6} \dot{X}$ & 77708.4 & -197007.5 & -115748.2 & -61.2 & -9.3 \\
$F_{7} \dot{Y}$ & - & - & $0.3 \times 10^{-4}$ & $-0.1 \times 10^{-7}$ & 5.5 \\
\hline$G_{1} \ddot{X}$ & -3071.9 & -4291.0 & -2.1 & -0.2 & $0.1 \times 10^{-4}$ \\
$G_{2} \ddot{Y}$ & - & $0.1 \times 10^{-9}$ & $-0.6 \times 10^{-13}$ & $0.2 \times 10^{-4}$ & $-0.1 \times 10^{-7}$ \\
$G_{3} \dot{X}^{2}$ & - & 0.2 & $-0.2 \times 10^{-3}$ & $-0.1 \times 10^{-3}$ & $0.9 \times 10^{-8}$ \\
$G_{4} \dot{Y}^{2}$ & - & - & - & $0.9 \times 10^{-4}$ & $-0.4 \times 10^{-7}$ \\
$G_{5} \dot{X} \dot{Y}$ & - & - & - & $0.5 \times 10^{-13}$ & $-0.5 \times 10^{-16}$ \\
$G_{6} \dot{X}$ & - & $-0.1 \times 10^{-2}$ & $0.9 \times 10^{-6}$ & -244.3 & 0.2 \\
$G_{7} \dot{Y}$ & - & 20120.4 & 1271.7 & -12.8 & $0.1 \times 10^{-9}$ \\
\hline
\end{tabular}

much less magnitude, being moved to the second member. This gives:

$\left\{\begin{array}{l}F_{2} \ddot{Y}+F_{6} \dot{X}=\frac{L}{A}-F_{1} \ddot{X}-F_{3} \dot{X}^{2}-F_{4} \dot{Y}^{2}-F_{5} \dot{X} \dot{Y}-F_{7} \dot{Y} \\ G_{1} \ddot{X}+G_{7} \dot{Y}=\frac{M}{A}-G_{2} \ddot{Y}-G_{3} \dot{X}^{2}-G_{4} \dot{Y}^{2}-G_{5} \dot{X} \dot{Y}-G_{6} \dot{X}\end{array}\right.$

The unknowns also appear in the second members of Eqs. (30), but according to Table 2, their effects are of the second order. Such a form of the equations facilitates finding the solution to a high degree of accuracy by successive approximations.

Each coefficient $F_{2}, F_{6}, G_{1}, G_{7}$, appearing in the first member of the equations, can be split into two parts, one prominent term, which is constant, and an additional term, of much less magnitude, denoted $F_{2}^{\prime}, F_{6}^{\prime}, G_{1}^{\prime}, G_{7}^{\prime}$, respectively which is dependent, at the second order, on the variables $X$ and $Y$. In a further step in transforming the equations into a form which facilitates finding a solution by successive approximations, the terms multiplied by $F_{2}^{\prime}, F_{6}^{\prime}, G_{1}^{\prime}, G_{7}^{\prime}$, can be moved to the second member; this allows us to write the equations as:

$$
\left\{\begin{array}{l}
-\ddot{Y}+\frac{C \Omega}{A} \dot{X}=\frac{L}{A}-F_{1} \ddot{X}-F_{2}^{\prime} \ddot{Y}-F_{3} \dot{X}^{2}-F_{4} \dot{Y}^{2} \\
-F_{5} \dot{X} \dot{Y}-F_{6}^{\prime} \dot{X}-F_{7} \dot{Y} \\
\ddot{X}+\frac{C \Omega}{A} \dot{Y}=\frac{M}{A}-G_{1}^{\prime} \ddot{X}-G_{2} \ddot{Y}-G_{3} \dot{X}^{2}-G_{4} \dot{Y}^{2} \\
-G_{5} \dot{X} \dot{Y}-G_{6} \dot{X}-G_{7}^{\prime} \dot{Y},
\end{array}\right.
$$

where:

$$
\begin{aligned}
& F_{2}^{\prime}=F_{2}+1 ; F_{6}^{\prime}=F_{6}-\frac{C \Omega}{A} ; \\
& G_{1}^{\prime}=G_{1}-1 ; G_{7}^{\prime}=G 7-\frac{C \Omega}{A} .
\end{aligned}
$$

This provides a form of the rigorous equations in the axial-symmetric case, that is suitable for a practical semianalytical integration. Using (27) and (28), we can see that the coefficients $F_{2}^{\prime}, F_{6}^{\prime}, G_{1}^{\prime}$ and $G_{7}^{\prime}$ are such that:

$F_{2}^{\prime} \simeq-\frac{1}{2} Y^{2}-\frac{3}{8} X^{2} Y^{2}$

$F_{6}^{\prime} \simeq \frac{C \Omega}{A}\left\{\frac{1}{2} X^{2}+\frac{3}{8} X^{4}+\frac{3}{8} X^{2} Y^{2}+\frac{5}{16} X^{6}\right\}$

$G_{1}^{\prime} \simeq \frac{1}{2} X^{2}+\frac{3}{8} X^{4}+\frac{3}{8} X^{2} Y^{2}+\frac{5}{16} X^{6}$

$G_{7}^{\prime} \simeq \frac{C \Omega}{A}\left\{\frac{1}{2} Y^{2}+\frac{3}{8} X^{2} Y^{2}\right\}$

which makes clear that the terms multiplied by these coefficients are of the second order in the $X, Y$ quantities.

\section{Methods for solving the equations in terms of $X$ and $Y$}

\subsection{Use of the intermediary variables $\varpi_{1}$ and $\varpi_{2}$}

One possibility for providing solutions for the $X$ and $Y$ variables in the axially symmetric case is to integrate Eqs. (18) to 
provide a solution for $\varpi_{1}$ and $\varpi_{2}$ and then to use relation (20) to obtain the solution for $X$ and $Y$.

Equations (18) can be transformed into the following form where the first members of the equations include only the largest terms as function of the variables $\varpi_{1}$ and $\varpi_{2}$, the other ones, which are much smaller, being moved into the second members:

$\left\{\begin{array}{l}\dot{\varpi}_{1}+\frac{C}{A} \Omega \varpi_{2}=\frac{L}{A}+\dot{s} \varpi_{2} \\ \dot{\varpi}_{2}-\frac{C}{A} \Omega \varpi_{1}=\frac{M}{A}-\dot{s} \varpi_{1} .\end{array}\right.$

These equations can be integrated using the method of "variations of parameters" (Moulton 1930) in a similar way than the method described in Woolard (1953) and in Bretagnon et al. (1997). This consists in using the solution for the reduced system as obtained when the second member is reduced to zero and to get a particular solution of the general equations as an expression with the same form, but with the constants of integration transformed into time-dependent quantities.

The reduced equations are:

$\left\{\begin{array}{l}\dot{\varpi}_{1}+\frac{C}{A} \Omega \varpi_{2}=0 \\ \dot{\varpi}_{2}-\frac{C}{A} \Omega \varpi_{1}=0\end{array}\right.$

of which the solution has the following form:

$\varpi_{1}=K_{\mathrm{s}} \sin \sigma t+K_{\mathrm{c}} \cos \sigma t$

$\varpi_{2}=K_{\mathrm{c}} \sin \sigma t-K_{\mathrm{s}} \cos \sigma t$

$K_{\mathrm{s}}$ and $K_{\mathrm{c}}$ being the constants of integration of the free motion and $\sigma$ its frequency (i.e. Euler frequency in the celestial system):

$\sigma=\frac{C}{A} \Omega$.

The solution of the general equations derived by the method of the variations of parameters has a form similar to (36), but with $K_{\mathrm{c}}$ and $K_{\mathrm{s}}$ considered as being time dependent quantities $K_{\mathrm{c}}(t)$ and $K_{\mathrm{s}}(t)$, such that $\varpi_{1}$ and $\varpi_{2}$ verify Eqs. (34). This gives:

$\dot{K}_{\mathrm{s}}=\frac{L}{A} \sin \sigma t-\frac{M}{A} \cos \sigma t+\dot{s}\left(\varpi_{2} \sin \sigma t+\varpi_{1} \cos \sigma t\right)$

$\dot{K}_{\mathrm{c}}=\frac{L}{A} \cos \sigma t+\frac{M}{A} \sin \sigma t+\dot{s}\left(\varpi_{2} \cos \sigma t-\varpi_{1} \sin \sigma t\right)$.

A quadrature of (38) will give the expression for $K_{\mathrm{c}}(t)$ and $K_{\mathrm{s}}(t)$, which once substituted into (36) will provide the expression as function of time for the intermediary variables $\varpi_{1}$ and $\varpi_{2}$.

Using relation (20) gives:

$\varpi_{1}=A_{1} \dot{X}+B_{1} \dot{Y}$

$\varpi_{2}=A_{2} \dot{X}+B_{2} \dot{Y}$.

or, equivalently:

$\dot{X}=a_{1} \varpi_{1}+b_{1} \varpi_{2}$

$\dot{Y}=a_{2} \varpi_{1}+b_{2} \varpi_{2}$, with:

$a_{1}=\frac{B_{2}}{A_{1} B_{2}-A_{2} B_{1}} ; b_{1}=\frac{-B_{1}}{A_{1} B_{2}-A_{2} B_{1}}$
$a_{2}=\frac{-A_{2}}{A_{1} B_{2}-A_{2} B_{1}} ; b_{2}=\frac{A_{1}}{A_{1} B_{2}-A_{2} B_{1}}$.

The development of the coefficients $a_{1}, a_{2}, b_{1}, b_{2}$ derived from a Taylor development of the quantities $Z$ and $s$ in terms of $X$ and $Y$ is:

$a_{1}=\frac{1}{2} X Y+\frac{5}{8} X^{3} Y+\frac{5}{8} X Y^{3}$

$b_{1}=1-\frac{1}{2} X^{2}-\frac{1}{8} X^{2} Y^{2}-\frac{1}{8} X^{4}$

$a_{2}=-1+\frac{1}{2} Y^{2}+\frac{1}{8} X^{2} Y^{2}$

$b_{2}=1+\frac{1}{2}\left(X^{2}+Y^{2}\right)+\frac{3}{8} X^{4}+\frac{11}{8} X^{2} Y^{2}$,

which allows us to derive the $X$ and $Y$ expressions from the developments obtained for $\varpi_{1}$ and $\varpi_{2}$ by a simple quadrature of (40) and successive approximations of the developments of expressions (42).

\subsection{Integration of the equations in the $X, Y$ variables}

Another possibility for providing solutions for the $X$ and $Y$ variables in the axially symmetric case is to directly integrate the equations in terms of these variables. The same method of variations of parameters as used for integrating Eqs. (34) can be employed for integrating Eqs. (31) that can also be written in a simpler form as:

$\left\{\begin{array}{l}-\ddot{Y}+\frac{C \Omega}{A} \dot{X}=\frac{L}{A}+F^{\prime \prime} \\ \ddot{X}+\frac{C \Omega}{A} \dot{Y}=\frac{M}{A}+G^{\prime \prime},\end{array}\right.$

with:

$F^{\prime \prime}=-F_{1} \ddot{X}-F_{2}^{\prime} \ddot{Y}-F_{3} \dot{X}^{2}-F_{4} \dot{Y}^{2}-F_{5} \dot{X} \dot{Y}-F_{6}^{\prime} \dot{X}-F_{7} \dot{Y}$

$G^{\prime \prime}=-G_{1}^{\prime} \ddot{X}-G_{2} \ddot{Y}-G_{3} \dot{X}^{2}-G_{4} \dot{Y}^{2}-G_{5} \dot{X} \dot{Y}-G_{6} \dot{X}-G_{7}^{\prime} \dot{Y}$.

The reduced equations are:

$\left\{\begin{array}{c}-\ddot{Y}+\frac{C \Omega}{A} \dot{X}=0 \\ \ddot{X}+\frac{C \Omega}{A} \dot{Y}=0\end{array}\right.$

of which the solution has the following form:

$\dot{X}=-K_{\mathrm{c}}^{\prime} \sin \sigma t+K_{\mathrm{s}}^{\prime} \cos \sigma t$

$\dot{Y}=K_{\mathrm{s}}^{\prime} \sin \sigma t+K_{\mathrm{c}}^{\prime} \cos \sigma t$

$K_{\mathrm{s}}^{\prime}$ and $K_{\mathrm{c}}^{\prime}$ being the constants of integration of the free motion with the Euler frequency $\sigma$ in the celestial system.

Note that, according to the CIP definition, the free motion itself will have to be taken into account as a motion in the ITRS, but in contrast, when solving the rotational equations in space, the resonance frequency of the reduced equations is the frequency of the free motion in the GCRS.

The general solution for $X$ and $Y$ is obtained by a quadrature of the expression for $\dot{X}$ and $\dot{Y}$, these latter quantities being 
obtained by substituting into (46), the expression derived from the computation of:

$$
\begin{aligned}
& \dot{K}_{\mathrm{s}}^{\prime}=-\frac{L}{A} \sin \sigma t+\frac{M}{A} \cos \sigma t-F^{\prime \prime} \sin \sigma t+G^{\prime \prime} \cos \sigma t \\
& \dot{K}_{\mathrm{c}}^{\prime}=-\frac{L}{A} \cos \sigma t-\frac{M}{A} \sin \sigma t-F^{\prime \prime} \cos \sigma t-G^{\prime \prime} \sin \sigma t
\end{aligned}
$$

where $F^{\prime \prime}$ and $G^{\prime \prime}$ are given by (44).

\section{The semi-analytical approach}

In order to obtain the solutions of the differential equations established in the previous sections, using, in the second members of these equations, the expressions for the perturbing torque exerted on the Earth, we will have to compute a number of sums, products, derivatives and integrations of semianalytical expressions. We will describe in this section the approach that has been followed for dealing with such series in order to obtain the solutions in a semi-analytical form; we will report on the evaluated efficiency of the integration process and on the main characteristics of the solutions.

\subsection{Fourier and Poisson series manipulations}

The semi-analytical computations performed in this paper as well as in the following one (Paper II) for (i) obtaining the expressions of the components of the external torque and (ii) solving the differential equations, are based on the software package GREGOIRE developed by J. Chapront (2003). More details on this software can be found in Capitaine et al. (2003).

All the semi-analytical expressions to be used include a polynomial form in time and a periodic part with several hundreds or thousands of Fourier and Poisson terms.

\subsection{Tests on the efficiency of the approach for integrating the equations}

The complete solution $X, Y$ of the equations of the rigid Earth corresponding to the expression of the perturbing torque derived from the theories of the Moon, the Sun and the planets will be computed in the next paper (Paper II) based on the equations established in this paper. Before performing such a complete computation, we have made a number of simulations for testing the efficiency of the approach.

The tests have used the GREGOIRE software and have been based on a "simulated" semi-analytical expression of the torque components corresponding to the IAU 2000 precessionnutation. These torque components have been derived from the rigorous rotational equations of the axially-symmetric rigid Earth, expressed in terms of Euler angles between the J2000 ecliptic system and the equatorial system of date (see for example Woolard 1953 or Bretagnon et al. 1997). The computation has consisted in writing that the second members of these two first equations, which are the two first torque components divided by $A$ (the Earth's equatorial moment of inertia), equal the first members of these equations, expressed in terms of the Euler angles $\psi, \omega$ and $\phi$, or equivalently, of the precession and nutation angles $\psi_{A}^{\prime}=\psi_{A}+\Delta \psi_{A}$ and $\omega_{A}^{\prime}=\omega_{A}+\Delta \omega_{A}$ in longitude and obliquity, respectively referred to the $\mathrm{J} 2000$ ecliptic (see Table 1). The IAU 2000 expressions of the torque components have thus been derived from the following equations using the IAU 2000A expressions of the precession-nutation quantities:

$$
\begin{aligned}
& \frac{L_{1}}{A}=-\ddot{\omega_{A}^{\prime}}+\sigma \dot{\psi}_{A}^{\prime} \sin \omega_{A}^{\prime}+\dot{\psi}_{A}^{\prime 2} \sin \omega_{A}^{\prime} \cos \omega_{A}^{\prime} \\
& \frac{M_{1}}{A}=\sin \omega_{A}^{\prime} \ddot{\psi_{A}^{\prime}}+\sigma \dot{\omega}_{A}^{\prime}+2 \dot{\psi_{A}^{\prime}} \dot{\omega}_{A}^{\prime} \cos \omega_{A}^{\prime} .
\end{aligned}
$$

Note that the torque components obtained in this way have no dynamical meaning, but are simply "simulated" expressions for testing the integration process. Note also that the components $L_{1}, M_{1}$ are referred to the CIP equator and to the equinox $\gamma_{1}$ to which the considered Euler angles actually refer (see Table 1 and Fig. 2). These components have thus to be converted into components $L, M$ in the CIRS' to be used as second members of Eqs. (34) or (43) to be solved for obtaining the $X, Y$ solutions.

The purpose of these simulations was to test and to compare the two methods described in Sects. 4.1 and 4.2, and also to evaluate the accuracy of the results. Such an evaluation was obtained by comparing the semi-analytical solution for $X, Y$ with the solution, used here as a reference, which was derived indirectly by Capitaine et al. (2003a) from the IAU 2000 solution for the classical quantities $\psi_{A}, \omega_{A}$ of precession and $\Delta \psi$ and $\Delta \varepsilon$ of nutation.

The simulations have consisted in:

(i) constructing the IAU 2000 semi-analytical expression of the "simulated" torque components $L_{1}, M_{1}$ following the approach described above (i.e. expressing the first members of the rotational equations in terms of the IAU 2000 expressions of the classical precession-nutation angles);

(ii) transforming the components $L_{1}, M_{1}$ into components $L, M$ in the CIRS' by applying the appropriate rotation (i.e. $E O-s-\chi_{A}-\Delta \chi_{A}$ about the CIP axis; see Fig. 2) to the semi-analytical series for the quantities $L_{1}$ and $M_{1}$;

(iii) integrating the rotational equations, using the two methods described in Sects. 4.1 and 4.2, respectively:

- Eqs. (34), and then (40);

- Eqs. (43);

doing successive approximations, starting from a very simplified expression of $X$ and $Y$, up to a convergence of the solution at a $0.01 \mu$ as level;

(iv) comparing solutions for $X, Y$ obtained by the two methods; (v) comparing the solutions for $X, Y$ with those derived indirectly from the IAU 2000 solutions for the classical precession-nutation variables.

The simplified expression to be used to begin the iteration process was a rough approximation of the linear terms of $X$ and $Y$, respectively. The obtained expressions were the sum of a polynomial form in $t$ of degree 5 and a sum of Fourier and Poisson terms having the arguments of the IAU 2000 nutation.

The numerical values for the phases and frequencies of the fundamental arguments of the series used in this preliminary 
step of the work have been those of the IAU 2000 nutation as provided in the IERS Conventions (2003) and the numerical value for the frequency of the free motion (37) of the Earth in the GCRS, $\sigma=(C / A) \Omega(\Omega$ being the mean Earth's angular velocity), which is a key quantity for integrating the equations, has been $\sigma=230877.2099 \mathrm{rad} / \mathrm{cy}$.

The solutions with a cut-off of $0.1 \mu$ as include a polynomial form in $t$ of degree 5, plus 1157 and 941 Fourier terms in $X$ and $Y$, respectively and 278 (315), 53 (43), 5 (6) Poisson terms of degrees 1, 2 and 3 , respectively for these two quantities.

The tests have shown that the approaches described in Sects. 4.1 and 4.2:

- are efficient in providing solutions for Earth rotation in terms of the $X, Y$ parameters that converges at a $0.01 \mu$ as level after only a very few iterations;

- provide independently $X, Y$ solutions that are consistent together within one microarcsecond, except for a few terms (see below).

Comparisons of the solution for the $X, Y$ quantities with respect to the IAU 2000 solutions obtained indirectly from the classical precession-nutation variables have shown that differences larger than $5 \mu$ as include:

- one constant term in each quantity;

- one linear term in $Y$ with an amplitude of $142 \mu \mathrm{as} / \mathrm{cy}$ and one quadratic term in $X$ with an amplitude of $1.6 \mu / \mathrm{cy}^{2}$;

- one term in each quantity with an amplitude larger than $200 \mu$ as corresponding to a nutation term with an amplitude of the order of 1 mas and a period greater than 100 centuries (i.e. argument: $2 l^{\prime}-2 F+2 D+2 \Omega$ );

- 17 terms with amplitudes larger than $5 \mu$ as all corresponding to very long periodic nutation terms with amplitudes of the order of a few tenths of mas.

This shows that there are no differences larger than $5 \mu$ as for $99 \%$ of the terms. Moreover the differences listed above can be explained as follows:

- the constants terms in the differences are due to the fact that the celestial pole offsets, which are part of the IAU $2000 X, Y$ expressions, are integration constants that cannot be determined by integration of the differential equations;

- the polynomial terms in the differences are due to the effect of the frame bias in right ascension (i.e. 14.6 mas) that is not present in the IAU 2000 expressions for the $\psi_{A}$ and $\omega_{A}$ quantities used for calculating the torque;

- the differences in the long periodic terms are due to the fact that the amplitudes of these terms are largely amplified by the integration process. Special care would have been required regarding the precision of the corresponding term of the torque before integration; this was not in the scope of this experimental stage based on a torque expression derived from the first and second order derivatives of the IAU 2000 precession-nutation.

Such tests have therefore validated the rotational equations established in this study, as well as the efficiency of the proposed approach and the integration process. The approach has been proved to be able to provide semi-analytical expressions of the solutions for the $X, Y$ variables with an accuracy better than $1 \mu$ as after one century when special care is taken for very long periodic terms. This will have to be especially taken into account in Paper II for the final step of the integration.

The method described in Sect. 4.2, which directly involves the $X, Y$ parameters, will be the method retained in Paper II for finding the complete solution. The method described in Sect. 4.1, which has the advantage of being based on a simpler form of the differential equations to be solved, can be considered only as being an intermediary step for comparison and validation purpose.

\subsection{Contributions to the solutions from the different terms of the equations}

In order to best understand the integration process in terms of the $X, Y$ variables, we have computed separately the contributions to the solution from:

- the solution of the simplified form of the equations $\dot{X}=\frac{L}{\sigma A}$ and $\dot{Y}=\frac{M}{\sigma A}$, similar to the so-called "Poisson equations" in the case of Euler angles (Woolard 1953);

- the complementary part $\mathrm{d} X=\frac{\dot{Y}}{\sigma}$ and $\mathrm{d} Y=\frac{-\dot{X}}{\sigma}$ of the solutions of Eqs. (43), similar to the so-called "Oppolzer terms" in the case of Euler angles (Woolard 1953);

- the solution of Eqs. (43) with the second member reduced to the $F^{\prime \prime}$ and $G^{\prime \prime}$ terms; such solutions represent the effects due to the deviation of the $X, Y$ variables from the simplest form of variables of this problem, such that $\psi_{A}^{\prime} \sin \epsilon_{0}$ and $\omega_{A}^{\prime}$.

This has shown where the main contributions to the solutions in $X, Y$ come from. The solution of the simplified form of the equations (i.e. Poisson equations) has obviously been found to be the major contribution. Additionally to this part, "Oppolzer terms" in $X$ and $Y$ consist of hundred of terms greater than $1 \mu \mathrm{as}$, whose largest ones are of the order of a few mas (Fourier terms) and a few hundred of $\mu$ as (Poisson terms). Finally, the contributions from $F^{\prime \prime}$ and $G^{\prime \prime}$ greater than $0.1 \mu$ as are composed of:

- polynomial terms of:

$$
\begin{aligned}
& -0{ }^{\prime} 031537 t^{3}+00^{\prime} 000020 t^{4} \text { in } X \text {; and } \\
& 00^{\prime} 0000004 t+0 \prime^{\prime} 000264 t^{4} \text { in } Y \text {; }
\end{aligned}
$$

- one Fourier term with an amplitude of $-0.4 \mu$ as in the $18.6 \mathrm{y}$ nutation in $Y$ and one Poisson term of degree 1 with an amplitude of $-12 \mu$ as;

- nine Poisson terms of degree 2 in $X$ with amplitudes larger than $1 \mu$ as (of which two of amplitudes larger than $20 \mu$ as) and one in $Y$ and two Poisson terms of degree 3 in $X$ and one in $Y$. 


\section{Expression of the equations for a deformable Earth}

In this section, we will study how the rotational equations for a rigid Earth in terms of the CIP $X, Y$ coordinates can be generalized to the case of a deformable Earth and how this would modify their solution. For this purpose, we will use Liouville equation (Munk \& MacDonald 1960) which relates the rotational angular momentum $\mathbf{I} \vec{\omega}$ with the torque $\vec{L}$ :

$\frac{\partial}{\partial t}(\mathbf{\Gamma} \vec{\omega})+\vec{\omega} \times(\mathbf{\Gamma})=\vec{L}$,

where $\mathbf{I}$ is the inertia tensor:

$\mathbf{I}=\left(\begin{array}{lll}A & F & E \\ F & B & D \\ E & D & C\end{array}\right)$

of which the elements are time-dependent, with $D, E$ and $F$ being small quantities whose squares, products and higher powers can be neglected.

\subsection{Rotational equations in the case of a deformable Earth}

Substituting (50) into (49) and retaining only the linear terms give the following expressions in terms of the parameters $\varepsilon$ and $\bar{A}$ :

$$
\left\{\begin{array}{l}
\bar{A} \dot{\omega}_{1}+(C-\bar{A}) \omega_{2} \omega_{3}-\varepsilon \bar{A}\left(\dot{\omega}_{1}+\omega_{2} \omega_{3}\right)+\dot{E} \omega_{3}-D \omega_{3}^{2}=\lambda \\
\bar{A} \dot{\omega}_{2}-(C-\bar{A}) \omega_{1} \omega_{3}+\varepsilon \bar{A}\left(\dot{\omega}_{2}-\omega_{1} \omega_{3}\right)+\dot{D} \omega_{3}+E \omega_{3}^{2}=\mu \\
C \dot{\omega}_{3}+\dot{C} \omega_{3}=v .
\end{array}\right.
$$

Similarly to the rigid case, we will limit the expressions to the axially symmetric case by neglecting the terms multiplied by the triaxiality factor $\varepsilon$ (which give rise only to the sub-diurnal nutations). This gives:

$$
\left\{\begin{array}{l}
\bar{A} \dot{\omega}_{1}+(C-\bar{A}) \omega_{2} \omega_{3}+\dot{E} \omega_{3}-D \omega_{3}^{2}=\lambda \\
\bar{A} \dot{\omega}_{2}-(C-\bar{A}) \omega_{1} \omega_{3}+\dot{D} \omega_{3}+E \omega_{3}^{2}=\mu \\
C \dot{\omega}_{3}+\dot{C} \omega_{3}=v .
\end{array}\right.
$$

We can express Eqs. (52) in terms of the intermediary variables $\varpi_{1}, \varpi_{2}$ and $\varpi_{3}$, using (14), with the simplification resulting from the rotational symmetry (i.e. $\Theta \approx \Omega-\dot{s}$ and $\bar{A}=A$ ). Substituting expression (20) of those variables in terms of $X$ and $Y$, we obtain the following system of differential equations for the case of a deformable body, corresponding to the two first equations of (52):

$$
\left\{\begin{array}{l}
F_{1} \ddot{X}+F_{2} \ddot{Y}+F_{3} \dot{X}^{2}+F_{4} \dot{Y}^{2}+F_{5} \dot{X} \dot{Y}+F_{6} \dot{X}+F_{7} \dot{Y}=\psi_{1} \\
G_{1} \ddot{X}+G_{2} \ddot{Y}+G_{3} \dot{X}^{2}+G_{4} \dot{Y}^{2}+G_{5} \dot{X} \dot{Y}+G_{6} \dot{X}+G_{7} \dot{Y}=\psi_{2},
\end{array}\right.
$$

where the functions $F_{i}$ and $G_{i}$ have the same expressions (25) and (26) as in the case of the rigid Earth and where,

$$
\begin{aligned}
\psi_{1}= & \frac{L}{A}-\frac{\Omega}{A}(\dot{E} \cos \Theta-\dot{D} \sin \Theta)+\frac{\Omega^{2}}{A}(D \cos \Theta+E \sin \Theta) \\
\psi_{2}= & \frac{M}{A}-\frac{\Omega}{A}(\dot{E} \sin \Theta+\dot{D} \cos \Theta), \\
& -\frac{\Omega^{2}}{A}(-D \sin \Theta+E \cos \Theta),
\end{aligned}
$$

are the components of the "excitation function": $\psi=\psi_{1}+i \psi_{2}$, classically split up (see, for example, Munk \& MacDonald (1960) or Moritz \& Mueller (1987)) into three components:

$\psi=\psi^{R D}+\psi^{L}+\psi^{\mathrm{TD}}$

with,

$\psi^{R D}$ being for the rotational deformation because of centrifugal distortions;

$\psi^{L}=\frac{L+i M}{A}$ for the external torque effect; and

$\psi^{\mathrm{TD}}$ for the tidal deformation of the Earth.

\subsection{Effect of the rotational deformation}

The periodic variations in the inertia tensor arising from the rotational deformation are given by (Munk \& MacDonald 1960, p. 25) as:

$D=\frac{k_{2}}{k_{\mathrm{s}}} \frac{C-A}{\Omega} \omega_{2}$

$E=\frac{k_{2}}{k_{\mathrm{s}}} \frac{C-A}{\Omega} \omega_{1}$

$F=0$,

where, $k_{2}$ is the "Love number" of degree 2 and $k_{\mathrm{s}}$ is the socalled "secular Love number".

Using expressions (56) in (54), allow us to write the part of the excitation function due to the rotational deformation in terms of $\varpi_{1}, \varpi_{2}, \dot{\varpi}_{1}, \dot{\varpi}_{2}$ and $\dot{s}$ as:

$\psi_{1}^{\mathrm{RD}}=\frac{k_{2}}{k_{\mathrm{s}}} \frac{C-A}{A}\left\{-\dot{\varpi}_{1}+\dot{s} \varpi_{2}\right\}$

$\psi_{2}^{\mathrm{RD}}=-\frac{k_{2}}{k_{\mathrm{s}}} \frac{C-A}{A}\left\{\dot{\varpi}_{2}+\dot{s} \varpi_{1}\right\}$,

which shows that the rotational deformation modifies Eqs. (34) by transforming the GCRS frequency of the free motion, $\sigma$, into the frequency $\Gamma$ :

$\Gamma=\frac{C \Omega}{A\left(1+\frac{k_{2}}{k_{\mathrm{s}}} \frac{C-A}{A}\right)} \simeq \sigma\left(1-\frac{k_{2}}{k_{\mathrm{s}}} \frac{C-A}{A}\right)$.

The numerical value of $\Gamma / \sigma$ corresponding to realistic values for the Love number (i.e. $k_{2} \approx 0.3 ; k_{\mathrm{s}} \approx 0.96$ and $\frac{C-A}{A} \approx 0.003$ ) is of the order of $1-0.001$.

The rotational equations in terms of $X$ and $Y$ should thus be written as:

$$
\left\{\begin{aligned}
F_{1} \ddot{X} & +F_{2} \ddot{Y}+F_{3} \dot{X}^{2}+F_{4} \dot{Y}^{2}+F_{5} \dot{X} \dot{Y}+F_{6}^{\prime \prime} \dot{X}+F_{7}^{\prime \prime} \dot{Y} \\
& =\psi_{1}^{L}+\psi_{1}^{\mathrm{TD}} \\
G_{1} \ddot{X} & +G_{2} \ddot{Y}+G_{3} \dot{X}^{2}+G_{4} \dot{Y}^{2}+G_{5} \dot{X} \dot{Y}+G_{6}^{\prime \prime} \dot{X}+G_{7}^{\prime \prime} \dot{Y} \\
& =\psi_{2}^{L}+\psi_{2}^{\mathrm{TD}}
\end{aligned}\right.
$$


where:

$$
\begin{aligned}
& F_{6}^{\prime \prime}=\Gamma \frac{1}{Z}\left\{1-a Y^{2}\right\}=\frac{\Gamma}{\sigma} F_{6} \\
& F_{7}^{\prime \prime}=\Gamma \frac{a}{Z} X Y=\frac{\Gamma}{\sigma} F_{7} \\
& G_{6}^{\prime \prime}=\Gamma \frac{a}{Z} X Y=F_{7}^{\prime \prime} \\
& G_{7}^{\prime \prime}=\Gamma \frac{1}{Z}\left\{1-a X^{2}\right\}=\frac{\Gamma}{\sigma} G_{7} .
\end{aligned}
$$

\section{3. $J_{2}$ rate contribution to the excitation function}

Considering a deformable Earth requires taking into account, in addition to the rigid Earth component $\psi^{L}=(L+i M) / A$ of the excitation function due to the external torque, the $J_{2}$ rate effect produced by the secular variation of the dynamical ellipticity of the Earth (see for example Williams 1994 or Bourda \& Capitaine 2004). $J_{2}$ is the zonal coefficient of the geopotential of degree 2, which is directly related to the dynamical ellipticity of the Earth, $H_{\mathrm{d}}$ (see for example Kinoshita 1977) by the relation: $J_{2} \approx \frac{C H_{\mathrm{d}}}{M R^{2}}, M$ being the mass of the Earth and $R$ the mean radius of the Earth.

This variation in $J_{2}$ (which is such that $\dot{J}_{2} / J_{2}$ is of the order of $-3 \times 10^{-6} / \mathrm{cy}$ ), creates a secular variation of the first order component of the external torque whose effect on precession was included in the recent $\mathrm{P} 03$ solution for precession (Capitaine et al. 2003b).

The additional contribution to $\psi^{L}$ due to the deformation of the Earth can be written, as:

$d \psi^{L}=\frac{\dot{J}_{2}}{J_{2}} t\left\{\frac{1}{A} \frac{3 G m}{a^{3}} M R^{2} J_{2}\left(\frac{a}{r}\right)^{3}\left(\begin{array}{c}\sin \Lambda \sin \varphi \cos \varphi \\ -\cos \Lambda \sin \varphi \cos \varphi \\ 0\end{array}\right)\right\}$

where the quantity between brackets is the first order part of the external torque acting on the Earth. In this expression, $G$ is the gravitational constant, $r, a$ and $m$ are the distance to the Earth, the mean distance and the mass of the perturbing body, respectively, $\Lambda, \varphi$, the ITRS longitude and latitude of this body.

Note that the additional effect $d \psi^{L}$ can equivalently be taken into account by multiplying the first order part of the torque by the factor: $\left(1+\dot{J}_{2} t / J_{2}\right)$.

\subsection{Effect of the tidal deformation of the Earth}

The effect of the tidal deformation of the Earth is to create the component $\psi^{\mathrm{TD}}$ of the excitation function. This component, which originates from the tidal potential, is the sum of terms appearing exactly at the same frequencies as the terms of the $\psi^{L}$ component due to the external torque. Considering the analytical expressions of these two parts of the excitation function as function of time, allows one to group the terms corresponding to the same frequency. This shows that the amplitude $A_{j}$ with frequency $\omega_{j}$ in the ITRS, of the term $j$ of the torque as expressed in the ITRS, is modified when considering the effect of $\psi^{\mathrm{TD}}$ in the following ratio:

$R_{j}=1-\frac{k_{2} n_{j}}{k_{\mathrm{s}} \Omega}$

$n_{j}=\Omega-\omega_{j}$, being the frequency of the term $\mathrm{j}$ in the GCRS.

\subsection{Change in the solution}

In the purpose of integrating the equations, it is convenient to rewrite (59) as follows:

$\left\{\begin{array}{l}-\ddot{Y}+\Gamma \dot{X}=\frac{L}{A}+d \psi_{1}^{L}+\psi_{1}^{\mathrm{TD}}+F^{\prime \prime} \\ \ddot{X}+\Gamma \dot{Y}=\frac{M}{A}+d \psi_{2}^{L}+\psi_{2}^{\mathrm{TD}}+G^{\prime \prime}\end{array}\right.$

The change in the Euler frequency, from $\sigma$ to $\Gamma$ given by (58), due to the elasticity of the Earth, will modify the expression for $K_{\mathrm{s}}^{\prime}(t)$ and $K_{\mathrm{c}}^{\prime}(t)$ derived by (38) and by (47), which, in turn, will modify the expression of the amplitudes of the periodic terms in $X$ and $Y$ (i.e. of the nutations). The effect will be dependent on the difference of the nutation frequency with respect to the frequency of the free motion.

The modifications of the amplitudes of nutation in $X$ and $Y$ due to the tidal effects will appear through a similar process than the previous effect (i.e. the corresponding change in $K_{\mathrm{s}}(t)$ and $K_{\mathrm{c}}(t)$ ), but in this case the change will be directly dependent on the nutation frequency.

The effect on the $X, Y$ solution of the $d \psi^{L}$ contribution due to the secular variation of the dynamical ellipticity of the Earth can be deduced from the $J_{2}$ rate effect on the precession and nutation angles computed by Capitaine et al. (2005a); this would correspond to a change of -3 mas $/ \mathrm{cy}^{2}$ in the quadratic term in $X$ and to the addition of a few Poisson terms in both coordinates $X$ and $Y$, the largest one being of the order of $20 \mu \mathrm{as} / \mathrm{cy}$ at the 18.6-yr period.

\section{Concluding remarks}

In this paper, we have established the equations of the rotational motion of the rigid Earth as function of the Earth Orientation Parameters (EOP) of the new paradigm that is recommended by the IAU 2000 resolutions to transform between the celestial and terrestrial reference systems. Starting from Euler dynamical equations for a rigid Earth, and using expressions for the components of the instantaneous rotation vector as functions of the celestial coordinates $X, Y$ of the Celestial intermediate pole, and the Earth rotation angle, ERA, the equations of Earth rotation were obtained explicitly in terms of $X, Y$ and the ERA. Then, taking into account the order of magnitude of the terms in these equations, we have developed the most appropriate form for a practical integration and we have investigated the methods for providing solutions for an axially symmetric Earth. We have performed a number of simulations for testing the approach and the integration process. These tests have shown that the method is efficient in providing semi-analytical solutions for $X$ and $Y$ with an accuracy compliant with that of the current IAU 2000A precession-nutation model. We have also provided the main characteristics of the solutions. Finally, 
we extended this approach to a model of a deformable Earth compliant with the $\mathrm{P} 03$ precession solution of Capitaine et al. (2003b) that includes the contribution of the secular variation of the dynamical ellipticity of the Earth.

This paper is the first in a series of papers describing the progress of the European DESCARTES Sub-project entitled "Advances in the integration of the equations of the Earth's rotation in the framework of the new parameters adopted by the IAU 2000 resolutions", which was described in Folgueira et al. (2004). The study presented here will be followed by another paper, denoted Paper II, providing the semi-analytical solutions of the equations (established in this paper) corresponding to the external torque derived from the most precise semi-analytical theories for the Moon, Sun and planets. This work is also accompanied by two other papers. One (Folgueira et al. 2005) is a review of the sets of variables to be used for a rigorous analytical integration of the equations of the Earth rotation. The other (Souchay et al. 2005) is for checking the solution against a numerical solution based on the same constants.

Acknowledgements. The research was carried out in the Department of "Systèmes de Référence Temps Espace" (SYRTE) of Observatoire de Paris and received financial support from Descartes Prize Allowance (2004) (M. Folgueira), for which we express our sincere appreciation. We are grateful to V. Dehant for her interest and encouragment and for helpful suggestions for improving the manuscript. We thank J. Chapront for having provided his software for the semi-analytical computations and for valuable discussion.

\section{References}

Bourda, G., \& Capitaine, N. 2004, A\&A, 428, 691

Bretagnon, P., Rocher, P., \& Simon, J.-L. 1997, A\&A, 319, 305

Brzezinski, A., \& Capitaine, N. 1993, J. Geophys. Res. 98(B4), 6667

Capitaine, N. 1990, Celest. Mech., 48, 127

Capitaine, N. 2000, in Towards Models and Constants for SubMicroarcsecond astrometry, ed. K. J. Johnston, D. D. McCarthy, B. Luzum, \& G. Kaplan, USNO, 153

Capitaine, N. 2002, in Proc. IERS Workshop, Paris, ed. N. Capitaine et al., Frankfurt am Main: Verlag des Bundesamts für Kartographie und Geodäsie, 35

Capitaine, N., Guinot, B., \& Souchay, J. 1986, Celest. Mech., 39, 283

Capitaine, N., Chapront, J., Lambert, S., \& Wallace, P. T. 2003a, A\&A, 400, 1145
Capitaine, N., Wallace, P. T., \& Chapront, J. 2003b, A\&A, 412, 567

Capitaine, N., Wallace, P. T., \& Chapront, J. 2005a, A\&A, 432, 355

Capitaine, N., Folgueira, M., \& Souchay, J. 2005b, Paper II, A\&A, submitted

Chapront, J. 2003, Notice, Paris Observatory (January 2003)

Folgueira, M., \& Souchay, J. 2005, A\&A, 432, 1101

Folgueira, M., Souchay, J., \& Capitaine, N. 2005, Celest. Mech., submitted

Folgueira, M., Capitaine, N., \& Souchay, J. 2004, in Proc. Journées 2004, Systèmes de Référence spatio-temporels, ed. N. Capitaine, Observatoire de Paris, 136

Guinot, B. 1979, in Time and the Earth's Rotation, ed. D. D. McCarthy, \& J. D. Pilkington (D. Reidel Publishing Company), 7

IAU NFA WG Recommendations 2005 (IAU Division 1 Working Group on "Nomenclature for Fundamental Astronomy"), http://syrte.obspm.fr/iauWGnfa/Explanatory.html, March 2005

IAU Transactions 2000, Vol. XXIVB; in Proc. Twenty-Fourth General Assembly; Manchester, ed. H. Rickman, ASP, Provo, USA, 2001, 34

IERS Conventions 2003, IERS Technical Note 32, ed. D. D. McCarthy, \& G. Petit, Frankfurt am Main: Verlag des Bundesamts für Kartographie und Geodäsie, 2004

Kinoshita, H. 1977, Celest. Mech., 15, 277

Kinoshita, H., Nakajima, K., Kubo, Y., et al. 1979, Publ. Int. Latitude Obs. Muzusawa, Vol. XII, N. 2, 71

Lieske, J. H., Lederle, T., Fricke, W., et al. 1977, A\&A, 58, 1

Loyer, S. 1997, Thèse (Ph.D.), Université Paul Sabatier, Toulouse III, France

Mathews, P. M., Herring, T. A., \& Buffett B. A. 2002, J. Geophys. Res. 107, B4, 10.1029/2001JB000390

Mathews, P. W. 2003, in Proc. Journées 2001, ed. N. Capitaine, Observatoire de Paris, 180

Moritz, H., \& Mueller, I. I. 1987, Earth rotation: Theory and Observation (New York: The Ungar Publishing Company)

Moulton, F. R. 1930, Differential equations, 2nd ed. (MacMillan Company)

Munk, W. H., \& MacDonald, G. J. F. 1960, The rotation of the Earth: A geophysical discussion (Cambridge University Press)

Roosbeek, F., \& Dehant, V. 1998, Celest. Mech., 70, 215

Souchay, J., Loysel, B., Kinoshita, H., \& Folgueira, M. 1999, A\&AS, 135,111

Souchay, J., Folgueira, M., \& Capitaine, N. 2005, A\&A, submitted

Williams, J. G. 1994, AJ, 108 (2), 711

Woolard, E. W. 1953, in Astronomical Papers prepared for the use of the American Ephemeris and Nautical Almanac, XV, Part I, 1 